\title{
Special Issue on Dynamic Traffic Assignment, Parts 1 and 2
}

\author{
Dynamic Traffic Assignment: Theory, Computation \\ and Emerging Paradigms
}

\section{Terry L. Friesz • Satish Ukkusuri}

Published online: 18 September 2014

(C) Springer Science+Business Media New York 2014

Dynamic traffic assignment (DTA) is now an established research specialty overlapping several fields of scholarly enquiry: civil engineering, industrial engineering, operations research, statistics, mathematics, computer science, regional science, city planning, complexity science, sustainability science, and economics. Several hundred scientists and engineers around the globe are devoting substantial energy to DTA research. Moreover, the theory and computational methods arising from DTA research continue to move closer to application and decision support in real-world environments.

In 2012 we issued a call for a broad spectrum of DTA research manuscripts in order to provide a snapshot in time of the diverse points of view being pursued by scholars around the world. Presenters at the Fourth International Symposium on Dynamic Traffic Assignment at Martha's Vineyard, USA, were invited to submit manuscripts for possible publication in Networks and Spatial Economics. From those invitations, we have assembled a collection of 29 papers, each of which has been comprehensively reviewed. Those 29 papers have been arranged as Parts I and II, which will appear under separate covers. Part I focuses on fundamental methodological advances in dynamic traffic assignment including network loading models, continuous time models, day to day dynamics. Part II focuses on application of dynamic traffic assignment in

T. L. Friesz ( $₫)$

Department of Industrial Engineering, Penn State, University Park, PA 16802, USA

e-mail: tlf13@engr.psu.edu

S. Ukkusuri

School of Civil Engineering, Purdue University, West Lafayette, IN, USA 
the context of transit networks, evacuation modeling and land use modeling. The following is a complete list of papers that comprise the DTA2012 Special Issue:

Part I:

(1) J. Ban: Convergence of Time Discretization Schemes for Continuous-Time Dynamic Network Loading Models

(2) J. Ban: Approximating Time Delays in Solving Continuous-Time Dynamic User Equilibria

(3) S. Boyles: A Continuous DUE Algorithm Using the Link Transmission Model

(4) G. E. Cantarella: Day-to-Day Dynamics \& Equilibrium Stability in a Two-Mode Transport System with Responsive Bus Operator Strategies

(5) M. Carey: Extending the Cell Transmission Model to Multiple Lanes and LaneChanging

(6) X. Di: On the Stability of a Boundedly Rational Day-to-day Dynamics

(7) S. Gao: Combining Disaggregate Route Choice Estimation with Aggregate Calibration of a Dynamic Traffic Assignment Model

(8) E. Ge: A Comparison of Dynamic User Optimal States with Zero, Fixed and Variable Tolerances

(9) S. Haji: Formulation of a Risk-Aver System Optimal Dynamic Traffic Assignment

(10) K. Han: Existence and Properties of State Operators in Dynamic User Equilibrium

(11) T. Iryo: Investigating Factors for Existence of Multiple Equilibria in Dynamic Traffic Network

(12) W. Jin: A New Relationship between Wardrop's User Equilibrium and Nash Equilibrium

(13) Khani: Trip-Based Path Algorithms Using the Transit Network Hierarchy

(14) M. Levin: Improving the Convergence of Simulation-Based Dynamic Traffic Assignment Methodologies

Part 2:

(15) J. Li: Evacuation Planning with Endogenous Transportation Network Degradations: A Stochastic Cell-based Model and Solution Procedure

(16) H. Lo: Combined Route Choice and Adaptive Traffic Control in a Day-to-day Dynamical System

(17) M. Mahut: An Efficient Method for Generating the Initial Path Set and Feasible Solution for Simulation-Based DTA

(18) M. Nie: A New Tradable Credit Scheme for the Morning Commute Problem

(19) H. Noh: Logit-based Congested Transit Assignment Using Hyperpaths on a Scheduled Transit Network

(20) S. Peeta: Advances in Dynamic Traffic Assgmnt: TAC Clearance Time Estimation for Incorporating Evacuation Risk in Routing Strategies for Evacuation Operations

(21) G. Ramadurai: A Case for Higher-order Traffic Flow Models in DTA

(22) W. Szeto: A Sustainable Road Network Design Problem with Land Use Transportation Interaction over Time 
(23) Sumalee: A Cell Transmission Model with Lane Changing and Incorporation of Stochastic Demand and Supply Uncertainties for Freeway Traffic State Estimation

(24) S. Travis Waller: Sustainability SI: Linear Programming Formulation for Strategic Dynamic Traffic Assignment

(25) V. Trozzi: Effects of Countdown Displays in Public Transport Route Choice Under Severe Overcrowding

(26) S. Ukkusuri: Integrated Agent-Based Dynamic Traffic Simulation Model for Hurricane Evacuation

(27) D. P. Watling: Model Representation \& Decision-Making in an Ever-Changing World: the Role of Stochastic Process Models of Transportation Systems

(28) Xie: An Excess-Demand Dynamic Traffic Assignment Approach for Inferring Origin-destination Demand Matrices

(29) W. F. Yushimito: Correcting the Market Failure in Work Trips with Work Rescheduling: an Analysis using Bi-level Models for the Firm-workers Interplay 